\title{
PROCESSING OF INTERSTELLAR DUST GRAINS IN GALAXIES
}

\author{
H. Kaneda ${ }^{1}$, D. Ishihara ${ }^{1}$, T. Onaka ${ }^{2}$, I. Sakon ${ }^{2}$, T. Suzuki ${ }^{3}$, K. Kobata ${ }^{1}$, \\ T. Kondo ${ }^{1}$, M. YAmagishi ${ }^{1}$, And A. Yasuda ${ }^{1}$ \\ ${ }^{1}$ Graduate School of Science, Nagoya University, Chikusa-ku, Nagoya 464-8602, Japan \\ ${ }^{2}$ Department of Astronomy, Graduate School of Science, University of Tokyo, Bunkyo-ku, Tokyo 113-0033, Japan \\ ${ }^{3}$ Institute of Space and Astronautical Science, Japan Aerospace Exploration Agency, Sagamihara, Kanagawa \\ 252-5210, Japan \\ E-mail: kaneda@u.phys.nagoya-u.ac.jp \\ (Received June 29, 2012; Accepted August 12, 2012)
}

\begin{abstract}
We have performed a systematic study of interstellar dust grains in various environments of galaxies. AKARI has revealed the detailed properties of dust grains not only in star-forming regions but also in regions not relevant to star formation, some of which are found not to follow our old empirical knowledge. Because of its unique capabilities, AKARI has provided new knowledge on the processing of large grains and polycyclic aromatic hydrocarbons (PAHs). For example, we detect PAHs from elliptical galaxies, which show unusual spectral features and spatial distributions, demonstrating importance of material processing in the interstellar space. We find that copious amounts of large grains and PAHs are flowing out of starburst galaxies by galactic superwinds, which are being shattered and destroyed in galactic haloes. We discover evidence for graphitization of carbonaceous grains near the center of our Galaxy, providing a clue to understanding the activity of the Galactic center. We review the results obtained from our AKARI program, focusing on the processing of carbonaceous grains in various environments of galaxies.
\end{abstract}

Key words: infrared: ISM; infrared: galaxies; ISM: dust, extinction; galaxies: ISM

\section{INTRODUCTION}

Many infrared (IR) observations have revealed that small grains including polycyclic aromatic hydrocarbons (PAHs) as well as large grains are ubiquitous and well mixed in the interstellar media (ISM) in our Galaxy and nearby galaxies. In particular, in starforming regions, dust and PAHs absorb stellar ultraviolet photons and re-radiate in the IR, where PAHs are responsible for $\sim 10 \%$ of the re-emitted IR fluxes. Hence the IR luminosities due to dust and PAH emission are both powerful tools to trace star-forming activities in galaxies. Beyond such star-formation tracers, however, spectral information on the dust and PAH emission would have much deeper physical implications for understanding the properties of the ISM.

We have performed a systematic study of interstellar dust grains in various environments of galaxies including our Galaxy, within the framework of the AKARI mission program, "ISM in our Galaxy and nearby galaxies" (Kaneda et al., 2009). AKARI has revealed the detailed properties of dust grains in regions not relevant to the star formation as well, some of which are found not to follow our old empirical knowledge. Because of its unique capabilities, such as near- and far-IR spectroscopy, the fine allocation of many photometric bands, and all-sky coverage, AKARI has provided new knowledge on the processing of dust and polycyclic aromatic hydrocarbons (PAHs) in the interstellar space.

The top panel in Fig. 1 shows the Galactic plane $9 \mu \mathrm{m}$ map of an area of about $175^{\circ} \times 60^{\circ}\left(l=-80^{\circ} \sim+95^{\circ}\right.$, $\left.|b|<30^{\circ}\right)$, which is created from the IRC all-sky survey data. The middle panel shows the $90 \mu \mathrm{m}$ map from the 

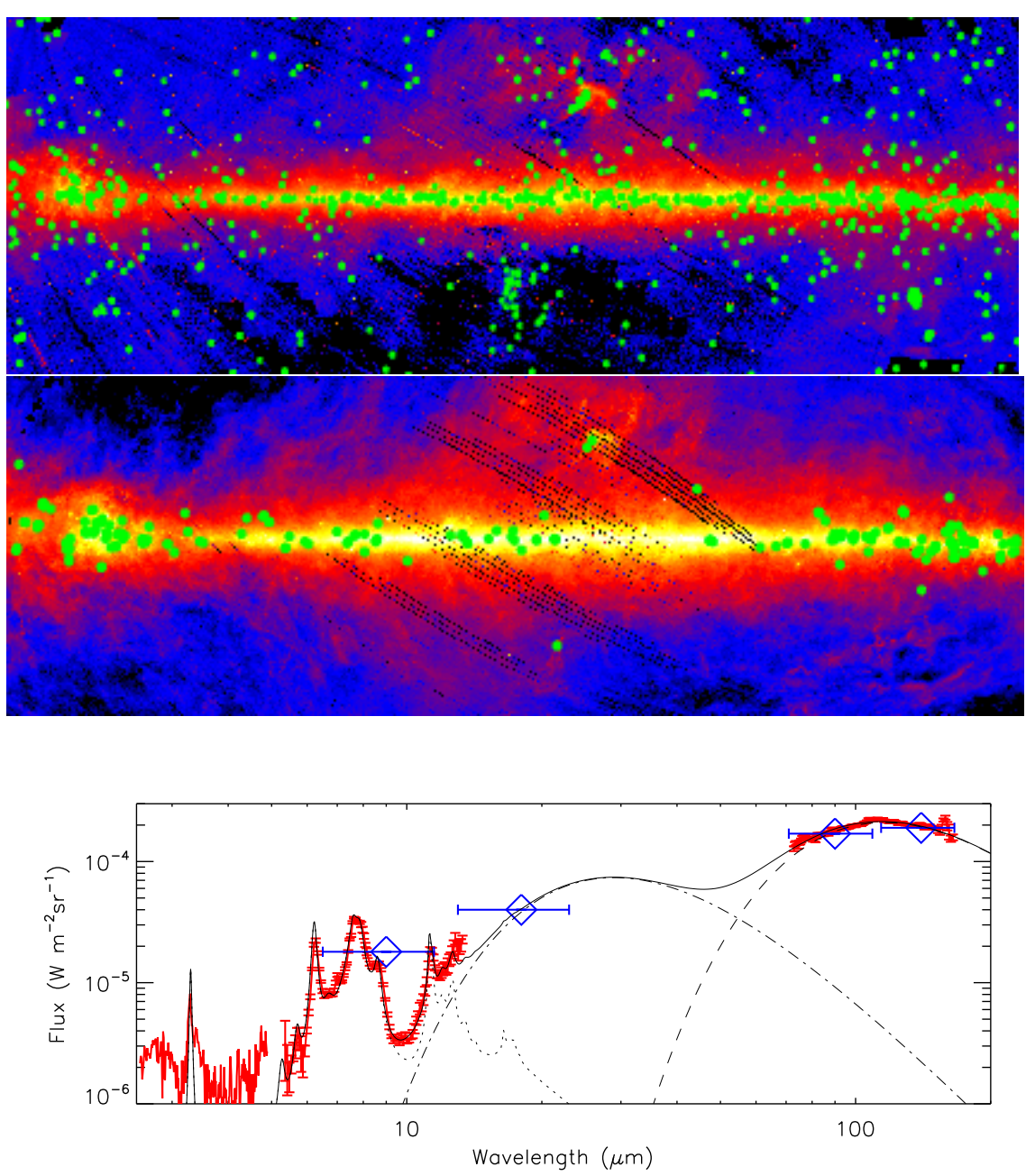

Fig. 1. (Top) Positions of spectroscopic observations with the IRC, overlaid on the Galactic plane $9 \mu \mathrm{m}$ map for an area of about $175^{\circ} \times 60^{\circ}\left(l=-80^{\circ} \sim+95^{\circ},|b|<30^{\circ}\right)$ created from the IRC all-sky survey data. (Middle) Positions of spectroscopic observations with the FIS, overlaid on the $90 \mu \mathrm{m}$ map from the FIS all-sky survey for the same area as the 9 $\mu \mathrm{m}$ map. (Bottom) Typical spectrum of the Galactic diffuse emission taken with the IRC at $2-13 \mu \mathrm{m}$ and the FIS at 70-160 $\mu \mathrm{m}$, along with the photometric data points at 9 and $18 \mu \mathrm{m}$ from the IRC and 90 and $140 \mu \mathrm{m}$ from the FIS all-sky survey. The curves represent an SED model consisting of the PAH, warm and cool dust components.

FIS all-sky survey for the same area as the $9 \mu \mathrm{m}$ map. The positions of spectroscopic observations with the IRC and FIS (FIS-FTS) are plotted on the $9 \mu \mathrm{m}$ and $90 \mu \mathrm{m}$ maps, respectively. The bottom panel shows a typical spectrum of the Galactic diffuse emission taken with the IRC at wavelengths of $2-13 \mu \mathrm{m}$ and the FIS at 70-160 $\mu \mathrm{m}$, along with the photometric data points at 9 and $18 \mu \mathrm{m}$ from the IRC and 90 and $140 \mu \mathrm{m}$ from the FIS all-sky survey. The figure clearly demostrates that a far-IR spectrum is almost feature-less while a near-to mid-IR spectrum is rich in features. The former is at- tributed to modified blackbody emission by large amorphous grains, while the latter is mostly PAH emission due to their discrete vibrational modes. We performed $\sim 600$ and $\sim 6,000$ spectroscopic observations at 70-160 $\mu \mathrm{m}$ and at $2-13 \mu \mathrm{m}$ or $2-5 \mu \mathrm{m}$ (Phase 3 ; warm mission phase), respectively. As can be seen in Fig. 1, AKARI reveals that PAHs are widely distributed, similarly to large grains; PAHs and large grains exhibit an excellent spatial correlation on various scales. The spectral properties of the ISM are also similar from region to region. However, in some specific interstellar conditions, 
we find that the situations are quite changeable, as will be shown below.

\section{ORIGINS OF DUST AND PAHs IN ELLIPTI- CAL GALAXIES}

It is known that the band ratios of the PAH emission features are almost the same among star-forming galaxies (Smith et al., 2007), indicating that the structures of PAHs are well organized and not disordered. However, with Spitzer and AKARI, we find that the PAH emission in elliptical galaxies exhibit unusual band ratios (Kaneda et al., 2008). Examples of AKARI 3-13 $\mu \mathrm{m}$ spectra of elliptical galaxies are displayed in Fig. 2, where the same diffuse ISM spectrum as in Fig. 1 is arbitrarily scaled and shown together for comparison. As can be seen in the figure, the usually strongest 7.7 $\mu \mathrm{m}$ feature is notably weak, whereas the $3.3 \mu \mathrm{m}$ and $11.3 \mu \mathrm{m}$ features are relatively strong. We concluded that neutral PAHs, rather than ionized ones, become dominant in very soft radiation field typical of ellitical galaxies, which causes the faint $\mathrm{C}-\mathrm{C}$ vibration features at 6-8 $\mu \mathrm{m}$. It should be noted that the PAH $3.3 \mu \mathrm{m}$ and $11.3 \mu \mathrm{m}$ emission does not represent any star-forming activity in this case. The AKARI detection of the PAH $3.3 \mu \mathrm{m}$ feature further implies that very small PAHs do exist, heated by the soft radiation causing the unusual faintness in the $7.7 \mu \mathrm{m}$ feature.

Fig. 2 shows strong absorption features around $4-5$ $\mu \mathrm{m}$. We identify them as blending of $\mathrm{SiO}(4.30 \mu \mathrm{m})$ and $\mathrm{CO}(4.66 \mu \mathrm{m})$ absorption due to K- and M-type O-rich stars (Mori et al., in this volume). The spectra also reveals a silicate emission feature around 10 $\mu \mathrm{m}$. Both of them suggest that silicate grains, but not carbonaceous grains, are currently supplied into the ISM by stellar mass loss from old stars. Then, where do the PAHs come from? Fig. 3 shows the spatial distributions of large grains and $\mathrm{PAHs}$ in the elliptical galaxies, NGC 4125 and NGC 4589. The distribution of the PAH $11.3 \mu \mathrm{m}$ emission is obtained with the Spitzer/IRS spectral mapping observations (Kaneda et al., 2011), while that of the dust emission is obtained by the AKARI/FIS slow-scan observations. The figure reveals that the PAHs exist only near the galactic centers, while the large grains are distributed much more widely, even considering the difference in the spatial resolution. PAHs must have been created by other process than the current stellar mass loss; one possibility is that they were reprocessed by fragmen-


Fig. 2. AKARI $3-13 \mu \mathrm{m}$ spectra of the elliptical galaxies, NGC 1316 and IC 3370, where stellar continuum components are subtracted. For comparison, the same diffuse ISM spectrum as in Fig.1 is shown in the red solid curve.

tation of larger carbonaceous grains near the centers. It seems, at least, that graphitized (aromatized) grains are relatively abundant near the galactic centers of the elliptical galaxies.

\section{STRUCTURAL CHANGES OF HYDROCAR- BONS IN SHOCKED REGIONS}

The properties of hydrocarbon grains can be probed by AKARI near-IR spectroscopy of the $3.3 \mu \mathrm{m}$ main feature and 3.4-3.6 $\mu \mathrm{m}$ sub-features. Both of them are attributed to the $\mathrm{C}-\mathrm{H}$ vibration mode of carbonaceous grains. The former is due to aromatic $\left(s p^{2}\right)$ hydrocarbons, while the latter is probably attributed to aliphatic $\left(s p^{3}\right)$ hydrocarbons (Duley \& Williams, 1981). They are likely to come from mixed aromatic-aliphatic organic nano-particles (Kwok \& Zhang, 2011). It has been believed that their intensity ratios do not vary much in the ISM. However AKARI reveals that they 


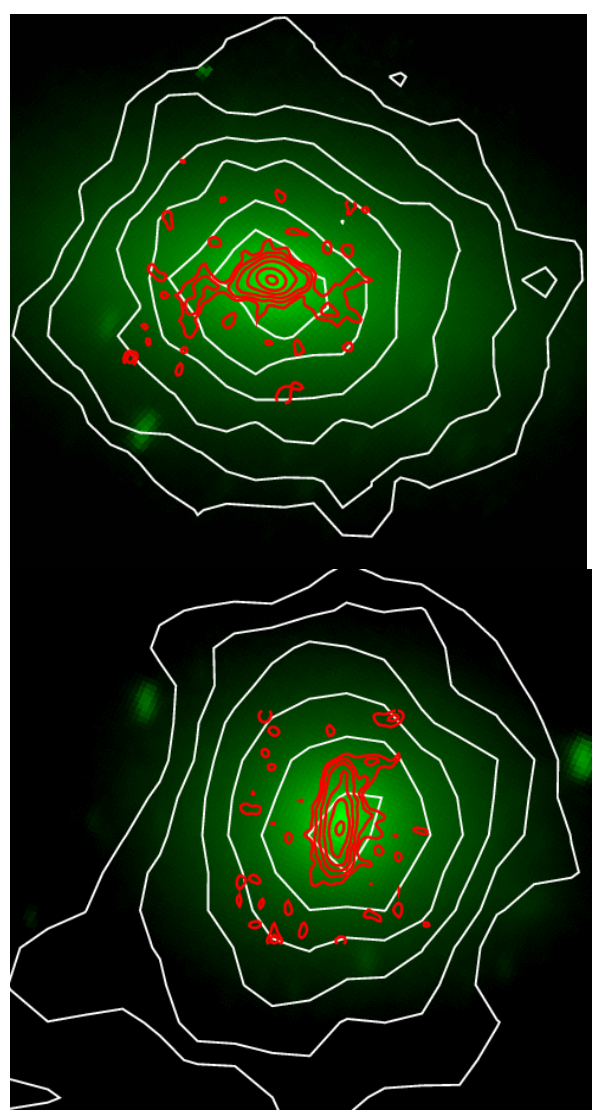

Fig. 3. AKARI $3 \mu \mathrm{m}$ images of (top) NGC 4125 and (bottom) NGC 4589 with the maps of the $90 \mu \mathrm{m}$ dust emission in the thin white contours and the PAH $11.3 \mu \mathrm{m}$ emission in the thick red contours. The sizes of the images are about $2^{\prime} .5 \times 2^{\prime} .5$. The contour levels are logarithmically spaced from 80 to $10 \%$ for the $90 \mu \mathrm{m}$ emission, while they are from 90 to $3 \%$ (NGC 4125) or 6\% (NGC 4589) for the $11.3 \mu \mathrm{m}$ emission.

considerably change, depending on interstellar conditions, which implies structural changes of the organic matter.

Fig. 4 shows AKARI 2.5-4.5 $\mu \mathrm{m}$ spectra of M82 for various regions including the center, disk, and halo regions. With AKARI, we find that copious amounts of large grains and PAHs are flowing out of the disk by galactic superwinds (Kaneda et al., 2010). With the near-IR spectroscopy, we clearly detect the PAH $3.3 \mu \mathrm{m}$ emission and the $3.4-3.6 \mu \mathrm{m}$ features in halo regions, which are located at a distance of $2 \mathrm{kpc}$ away from the galactic center, thus confirming the presence of very small PAHs even in the harsh environment of the M82


Fig. 4. AKARI 2.5-4.5 $\mu \mathrm{m}$ spectra of M82 from the center, disk, southern halo, and northern halo region from top to bottom (Yamagishi et al., 2012).

halo (Yamagishi et al., 2012). Similar results are also obtained for a filamentary outflow of NGC 1569 (Onaka et al., 2010). However the observed spectral properties are quite different from those commonly understood. In comparison with the spectra of the galactic center and disk regions (Fig. 4) as well as the spectrum of the diffuse ISM (Fig. 1), the 3.4-3.6 $\mu \mathrm{m}$ features are unusually abundant in the halo, suggesting the dominance of 
aliphatic structures over aromatic ones by shattering of hydrogenated amorphous carbon grains in shocks.

Similar results, i.e., notably strong aliphatic features, have so far been obtained for the foot points of CO molecular loops near the Galactic center (Kaneda et al., 2012a) and the inner bar connecting the starburst ring and the central AGN in NGC 1097 (Kondo et al., 2012). The CO molecular loops were found by NANTEN; Fukui et al. (2006) suggested that the molecular loops were formed by the buoyant rise of magnetic loops due to magnetic flotation driven by the Parker instability. Our result calls for such a condition in the magnetic flotation picture that the falling speed of the gas along the loop should be fast enough to alter the properties of hydrocarbon grains by shocks at its foot points. The detection of the strong aliphatic emission from the inner bar of NGC 1097 indicates that the gas and dust in the bar are in a turbulent motion, likely fueling the central AGN from the starburst ring.

\section{GRAPHITIZATION OF DUST IN THE GALAC- TIC CENTER}

AKARI is capable of far-IR $(70-160 \mu \mathrm{m})$ spectral mapping with FIS-FTS, thereby we simultaneously obtained the line and continuum maps in spatially and spectrally continuous ways, which contain important information about the ISM. For the Galactic center, we investigated spatial variations in the spectral shapes, focusing on dust continuum emission (Kaneda et al., 2012b). For this purpose, we reduced the spectral resolution to increase the signal-to-noise ratio of the dust continuum emission. The spectral fitting shows that two modified blackbody components with temperatures of $\sim 20 \mathrm{~K}$ and $\sim 50 \mathrm{~K}$ can reproduce most of the continuum spectra. For some spectra, however, we find that there is a significant excess on top of a modified blackbody continuum around 110-130 $\mu \mathrm{m}$ (Fig. 5). The excess emission probably represents a dust feature, which is found to be spatially correlated with the $\mathrm{OH}$ absorption and a CO cloud. Possible origins of the dust feature were discussed in Kaneda et al. (2012b): carbon onions or micron-sized graphite grains, based on graphite materials. We find that the dust model including the large graphite grains can at least reproduce the observed spectrum with the dust feature fairly well. The detection of the excess feature from this region might be related to the (past) activity of the Galactic center, because intense UV heating is needed to
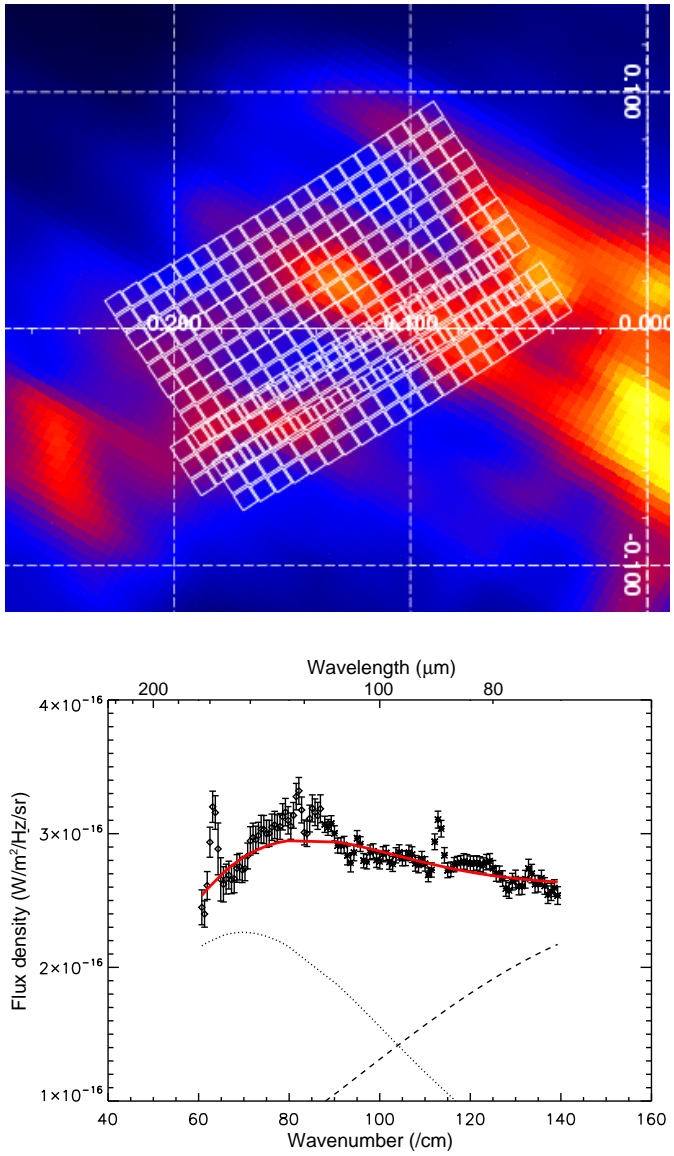

Fig. 5. (Top) Area of the Galactic center mapped by the spectroscopic observations with the FIS array, overlaid on the $90 \mu \mathrm{m}$ all-sky map in Galactic coordinates (same source as in Fig. 1). The field of view of each pixel is shown in the small box. (Bottom) FIS-FTS spectrum of the region toward the Arches cluster $\left(l \simeq 0^{\circ} .12, b \simeq 0^{\circ} .02\right)$. The result of the two-temperature modified blackbody model fitting is shown together, where the wavenumber ranges corresponding to the emission lines ([OIII], [NII], and [CII]) and the excess feature (80 to $90 \mathrm{~cm}^{-1}$ ) are masked in the fitting.

graphitize carbonaceous grains. This result may also be related with the abundant PAHs in the centers of elliptical galaxies as mentioned above, because many elliptical galaxies are known to possess galactic nuclei with declining or ceased activity like our Galaxy.

\section{SUMMARY}

AKARI has revealed various phenomena of processing of carbonaceous grains in harsh environments of galaxies. From elliptical galaxies, we detect unusual spectral features and spatial distributions of PAHs. PAHs 
exist only near the galactic centers, while large grains are distributed widely. The large grains are probably of stellar mass loss origin and mostly made of silicate. The PAHs must have been created by other process such as fragmentation of larger carbonaceous grains near the centers. From the galactic superwind of M82, the inner bar of NGC 1097, and the foot points of molecular loops near the Galactic center, we detect unusually strong aliphatic features at $3.4-3.6 \mu \mathrm{m}$ relative to the $3.3 \mu \mathrm{m}$ aromatic feature, which suggests shattering of carbonaceous grains by shocks. From the Galactic center, we detect a far-IR dust feature on top of a modified blackbody continuum around 110-130 $\mu \mathrm{m}$. We discuss possible origins of the dust feature based on graphite materials, i.e., carbon onions and micron-sized graphite grains. The detection of the dust feature from this region might be related to the (past) activity of the Galactic center.

\section{ACKNOWLEDGEMENTS}

This research is supported by a Grant-in-Aid for Scientific Research No. 22340043 from the Japan Society for the Promotion of Science, and the Nagoya University Global COE Program, "Quest for Fundamental Principles in the Universe: from Particles to the Solar System and the Cosmos", from the Ministry of Education, Culture, Sports, Science and Technology of Japan.

\section{REFERENCES}

Duley, W. W. \& Williams, D. A., 1981, The Infrared Spectrum of Interstellar Dust - Surface Functional Groups on Carbon, MNRAS, 196, 269

Fukui, Y., et al., 2006, Molecular Loops in the Galactic Center: Evidence for Magnetic Flotation, Science, 314, 106

Kaneda, H., Koo, B.-C., Onaka, T., \& Takahashi, H., 2009, AKARI Observations of the ISM in Our Galaxy and Nearby Galaxies, Adv. Sp. Res., 44, 1038

Kaneda, H., Onaka, T., Sakon, I., Kitayama, T., Okada, Y., \& Suzuki, T., 2008, Properties of Polycyclic Aromatic Hydrocarbons in Local Elliptical Galaxies Revealed by the Infrared Spectrograph on Spitzer, ApJ, 684, 270

Kaneda, H., et al., 2010, Large-Scale Distributions of Mid- and Far-Infrared Emission from the Center to the Halo of M82 Revealed with AKARI, A\&A, 514, A14
Kaneda, H., et al., 2011, Properties of Dust and PAHs in the Hot Plasma of the Elliptical Galaxy NGC 4125 Revealed with AKARI and Spitzer Space Telescope, PASJ, 63, 601

Kaneda, H., et al., 2012a, Processing of Polycyclic Aromatic Hydrocarbons in Molecular-Loop Regions Near the Galactic Center Revealed by AKARI, PASJ, 64, 25

Kaneda, H., et al., 2012b, Properties of Dust in the Galactic Center Region Probed by AKARI FarInfrared Spectral Mapping - Detection of a Dust Feature, arXiv:1205.4078

Kondo, T., et al., 2012, The Central Region of the Barred Spiral Galaxy NGC 1097 Probed by AKARI Near-infrared Spectroscopy, ApJ, 751, L18

Onaka, T., Matsumoto, H., Sakon, I., \& Kaneda, H., 2010, Detection of Unidentified Infrared Bands in a Halpha; Filament in the Dwarf Galaxy NGC 1569 with AKARI, A\&A, 514, A15

Kwok, S. \& Zhang, Y., 2011, Mixed AromaticAliphatic Organic Nanoparticles as Carriers of Unidentified Infrared Emission Features, Nature, 479,80

Smith, J. D. T., et al., 2007, The Mid-Infrared Spectrum of Star-Forming Galaxies: Global Properties of Polycyclic Aromatic Hydrocarbon Emission, ApJ, 656, 770

Yamagishi, M., et al., 2012, AKARI Near-Infrared Spectroscopy of the Aromatic and Aliphatic Hydrocarbon Emission Features in the Galactic Superwind of M82, A\&A, 541, 10 